\title{
Angular dependence of the magnetic susceptibility in the itinerant metamagnet $\mathrm{Sr}_{3} \mathrm{Ru}_{2} \mathrm{O}_{7}$
}

\author{
S. A. Grigera, ${ }^{1,2}$ R. A. Borzi, ${ }^{1}$ S. R. Julian, ${ }^{3}$ R. S. Perry,${ }^{4}$ Y. Maeno, ${ }^{4,5}$ and A. P. Mackenzie ${ }^{1}$ \\ ${ }^{1}$ School of Physics and Astronomy, University of St. Andrews, St. Andrews KY16 9SS, UK. \\ ${ }^{2}$ School of Physics and Astronomy, University of Birmingham, Birmingham B15 2TT, UK. \\ ${ }^{3}$ Cavendish Laboratory, Madingley Road, Cambridge CB3 OHE, UK. \\ ${ }^{4}$ Department of Physics, Kyoto University, Kyoto 606-8502, Japan. \\ ${ }^{5}$ International Innovation Centre, Kyoto University, Kyoto 606-8501, Japan.
}

(Dated: October 31, 2018)

\begin{abstract}
We report the results of a study of the differential magnetic susceptibility of $\mathrm{Sr}_{3} \mathrm{Ru}_{2} \mathrm{O}_{7}$ as a function of temperature and magnetic fields applied at a series of angles to the $a b$ plane. By analysing the real and imaginary parts of the susceptibility, we conclude that the field angle acts as a continuous tuning parameter for the critical end-point to a line of first order metamagnetic phase transitions. The end-point sits at approximately $1.25 \mathrm{~K}$ for fields applied in the $a b$ plane, and is depressed to below $50 \mathrm{mK}$ when the field is aligned within $10^{\circ}$ of the $c$ axis.
\end{abstract}

\section{INTRODUCTION}

Understanding unusual behaviour of itinerant carriers in correlated electron systems continues to be a major challenge of modern condensed matter physics. One of the most promising approaches is based on the concept of quantum criticality, in which many of the metallic properties are dominated by fluctuations associated with the presence of a $T \rightarrow 0$ critical point ${ }^{1.2 .3 .4 .5}$. Usually, quantum critical points result from the tuning of second order phase transitions. Recently, however, we have discussed the possibility that signatures of quantum criticality observed in the bilayer ruthenate $\mathrm{Sr}_{3} \mathrm{Ru}_{2} \mathrm{O}_{7}$ are associated with a $T \rightarrow 0$ critical end-point of a line of first-order metamagnetic phase transitions 6.7.8.9. The data on which this analysis was based were taken with the field aligned with the crystallographic $c$-axis, and it was argued that in this circumstance the critical end-point existed naturally at or very near $T=0$. However, no explicit evidence had been obtained for a key feature of the underlying picture, namely continuous tuning of a line of end-points terminating a surface of first-order transitions. In this paper we report a study of the angular dependence of the differential magnetic susceptibility, in which we demonstrate the existence of such a surface.

The most general definition of metamagnetism is a superlinear rise in magnetisation over a narrow range of applied magnetic field. This can occur in several circumstances. The term was first used in the context of antiferromagnetic insulators, for which the change in magnetisation is due to field-induced 'spin-flip' or 'spin-flop' transitions ${ }^{10}$. A form of metamagnetism more relevant to itinerant systems was first described by Wohlfarth and Rhodes ${ }^{11}$, who pointed out that strongly enhanced paramagnets on the verge of ferromagnetism might undergo a phase transition involving exchange splitting of the Fermi surface at some finite applied magnetic field. A characteristic of such systems is a strongly enhanced param- agnetic susceptibility with a pronounced maximum as a function of temperature, indicating the presence of a peak in the density of states very near the Fermi level. In a mean field description the application of a field leads to the Stoner criterion for exchange splitting being satisfied, resulting in a metamagnetic phase transition.

An itinerant electron metamagnetic phase transition of the kind described above is usually first order, because the presence of the magnetic field prevents a symmetry change - the Fermi surface becomes more polarised rather than spontaneously polarised ${ }^{12}$. These first order transitions have been seen in a number of systems, as described in a recent review by Goto, Fukamichi and Yamada 13 . In some cases $\left(\right.$ e.g. $\mathrm{Co}\left(\mathrm{S}_{1-x} \mathrm{Se}_{x}\right)_{2}$, for $x<0.1$ and $\mathrm{La}\left(\mathrm{Fe}_{1-x} \mathrm{Si}_{x}\right)_{13}$, for $\left.0.11<x<0.14\right)$, the systems undergo first order ferromagnetic phase transitions below some temperature $T_{c}$. As the temperature is raised higher than $T_{c}$, the ferromagnetic transition at zero field is changed to a first order metamagnetic transition. In others (e.g. $\mathrm{Co}\left(\mathrm{S}_{1-x} \mathrm{Se}_{x}\right)_{2}$, for $\left.x \geq 0.12\right)$, no ferromagnetism is seen, and first-order metamagnetic transitions are observed as $T \rightarrow 0$. In common with other first-order phase transitions, metamagnetic transitions change from being first order to being crossovers at sufficiently high temperature, with a critical end-point separating the two regimes. This end-point occurs at fairly high temperatures in some of the best-studied systems (e.g. $\approx 80 \mathrm{~K}$ in $\mathrm{Co}\left(\mathrm{S}_{1-x} \mathrm{Se}_{x}\right)_{2}$, for $x=0.14$, and $\approx 15 \mathrm{~K}$ in $\left.\mathrm{UCoAl}\right)$. It can be forced down in temperature by the application of hydrostatic pressure (to below 10K in UCoAl at $1.2 \mathrm{GPa})^{14}$, but to our knowledge, it has not been tuned to the $T \rightarrow 0$ in any metamagnet studied so far under pressure. However, any material has a natural effective pressure related to its electronic structure. In some materials metamagnetic crossovers rather than phase transitions are seen, even at low temperatures ${ }^{15}$ suggesting that chemical pressure is capable of depressing the endpoint to 'negative temperature'. 
The above discussion raises the possibility that in some circumstances, the chemical pressure of a material may be suitable to tune a metamagnetic end-point to, or very close to, $T=0$. On the basis of observations of transport properties, we have argued that $\mathrm{Sr}_{3} \mathrm{Ru}_{2} \mathrm{O}_{7}$ with the field aligned along the $c$ axis is an example of such a situation. If correct, this analysis suggests that the material displays an unusual form of quantum criticality without a symmetry-broken phase ${ }^{7.8}$. This would be an important development in the on-going quest to understand quantum critical points, so it is important to investigate the metamagnetism of $\mathrm{Sr}_{3} \mathrm{Ru}_{2} \mathrm{O}_{7}$ in more depth. Of particular interest would be the identification of a tuning parameter that would allow the identification of first-order transitions terminating in a finite-temperature critical point that could then be tuned smoothly to $T=0$. In the work reported to date, the criticality has been ascribed to such a non-symmetry breaking critical point, but because it already sits at $T=0$, no associated first order transition has been explicitly seen.

Experience on other itinerant metamagnets shows that pressure increases the characteristic field of the metamagnetic transition while at the same time depressing the end-point temperature $T^{*}$ (see ref 13). For the field aligned parallel to the $c$ axis of $\mathrm{Sr}_{3} \mathrm{Ru}_{2} \mathrm{O}_{7}$, pressure is therefore likely to favour a crossover rather than a first-order transition with a finite temperature end-point. However, the natural anisotropy of the metamagnetism suggests another route to achieving the desired tuning. The metamagnetic fields are slightly different for the two field orientations ( $5 \mathrm{~T}$ for $H \| a b$ and $7.8 \mathrm{~T}$ for $H \| c)^{6}$, but this is not the only feature of the anisotropy. The low temperature magnetoresistance is qualitatively different for the two field orientations, giving rise to the suspicion that for $H \| a b$, the transition may be first order at $T=0$. In a recent paper, Chiao et al ${ }^{16}$ have argued that measurements of the differential magnetic susceptibility for $H \| a b$ support the hypothesis of a first-order transition.

Here, we report the results of a detailed study of the differential magnetic susceptibility as a function of temperature, magnetic field and magnetic field angle. We combine observations of the real and imaginary parts of the susceptibility and of hysteresis to present strong evidence that the phase diagram contains a surface of firstorder phase transitions in $(H, T, \theta)$ space. The line of critical end-points that terminates this surface is tuned continuously towards zero temperature as the field is rotated away from the plane, falling below $50 \mathrm{mK}$ (the lowest temperature studied) when the angle is within $10^{\circ}$ of the $c$ axis. We further demonstrate that even the relatively low frequency ( $\approx 80 \mathrm{~Hz}$ ) employed for these a.c. measurements is not a good approximation to the static limit. Care must therefore be taken before drawing conclusions on the nature of the critical point from the field and temperature dependence of the susceptibility in its vicinity.

\section{EXPERIMENTAL}

The experiments described here were performed in dilution refrigerators in Cambridge and St. Andrews. The sample studied was a small piece of approximate dimension $(1 \times 1.1 \times 0.6) \mathrm{mm}^{3}$ cut from a single crystal of $\mathrm{Sr}_{3} \mathrm{Ru}_{2} \mathrm{O}_{7}$ grown in Kyoto whose residual resistivity had been measured to be $2.4 \mu \Omega \mathrm{cm}$. It was thermally grounded to the mixing chamber by electrical coupling through gold and copper wires. In order to work at temperatures above $1.1 \mathrm{~K}$ the normal regime of operation of the dilution fridge was changed by varying the ${ }^{3} \mathrm{He} /{ }^{4} \mathrm{He}$ mixture composition. The differential susceptibility was measured using a drive field of $3.3 \times 10^{-5} \mathrm{~T}$ r.m.s., and counterwound pickup coils each consisting of approximately 1000 turns of $12 \mu \mathrm{m}$ diameter copper wire. For most of the experiments, an excitation frequency of $79.96 \mathrm{~Hz}$ was employed. Low temperature transformers mounted on the $1 \mathrm{~K}$ pot of the dilution refrigerators were used throughout to provide an initial signal boost of approximately a factor of 100 . When the frequency dependence was studied during the later stages of the project, the frequency dependence of the transformer was explicitly calibrated rather than relying on generic performance data. This ensures that we have confidence in the relative magnitude of the signals presented here.

Direct measurement of the empty-coil signal as a function of angle established that at all angles studied, the background varied smoothly with field through the field region of interest (between 4.5 and 8.5 tesla). The data shown in Figs. 1-3 were obtained by subtracting a second order polynomial which modelled the combination of the empty coil background and the weakly varying nonmetamagnetic susceptibility from the raw signal. The data presented are therefore the extra susceptibility due to the metamagnetism of $\mathrm{Sr}_{3} \mathrm{Ru}_{2} \mathrm{O}_{7}$.

The absolute magnitude was more difficult to measure with high accuracy. It was calibrated by measuring the signal with and without the sample at fields between 1 and $3 \mathrm{~T}$ applied parallel to the $a b$ plane. The direct background subtraction gave the expected field-independent susceptibility in this field range, and this was calibrated against the known value ${ }^{6,17}$. The dilution refrigerator had to be removed from and then replaced in the cryostat in order to perform the empty run. The accuracy of our absolute calibration depends on the stability of the background signal throughout this process, and on the assumption that a measurement at approximately $80 \mathrm{~Hz}$ is equivalent to one at quasi-d.c. in this field range. However, we stress again that these concerns do not apply to the relative signal magnitudes that we report.

\section{RESULTS}

A representative sample of the large data set acquired during the course of this work is shown in Fig. 1. The real part of the dynamic susceptibility is plotted as a 


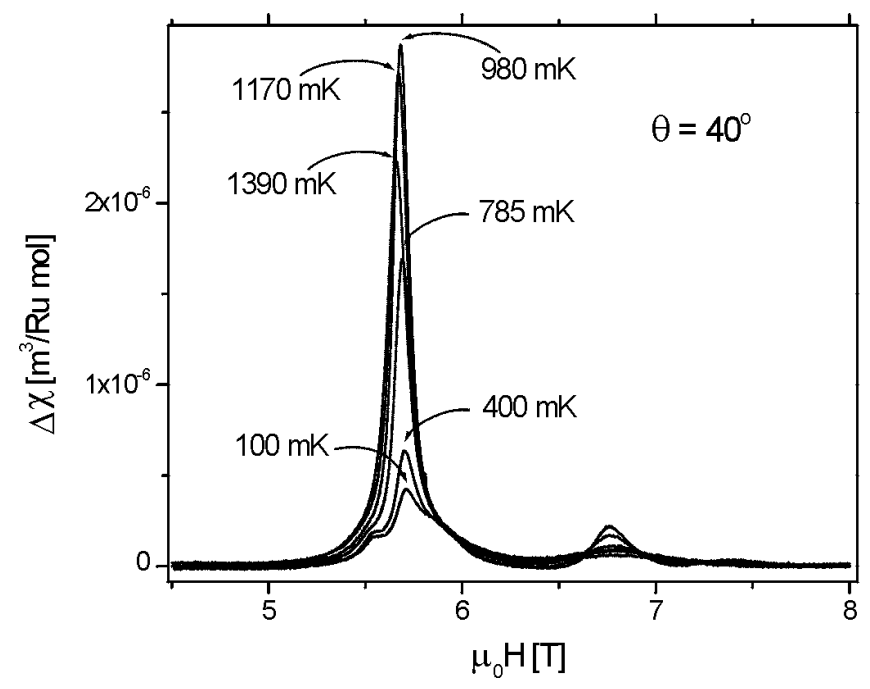

FIG. 1: The real part of the differential magnetic susceptibility through the metamagnetic transition in $\mathrm{Sr}_{3} \mathrm{Ru}_{2} \mathrm{O}_{7}$. A small oscillating field $\left(3.3 \times 10^{-5}\right.$ T r.m.s., $\left.79.96 \mathrm{~Hz}\right)$ is combined with a swept d.c. field, applied in this case at $40^{\circ}$ to the $a b$ plane. The main peak shows a pronounced maximum at approximately $1 \mathrm{~K}$, falling away in magnitude for temperatures higher or lower than this. All data were taken in an increasing d.c. field. The data are quoted as $\Delta \chi$ because of the background subtraction procedure described in the text.

function of a swept d.c. field applied at an angle of $40^{\circ}$ to the $a b$ plane. At this field angle, the data contain a main peak at approximately $5.7 \mathrm{~T}$, and a much weaker second peak at approximately $6.7 \mathrm{~T}$. The second peak washes out as the temperature is increased from $100 \mathrm{mK}$. This and a further minor peak exist when the field is applied parallel to the $a b$-plane, but both disappear rapidly with temperature and increasing field angle. Here, we concentrate our attention on the major peak. This peak grows as the temperature is increased from 100 $\mathrm{mK}$, reaching a pronounced maximum at approximately $1 \mathrm{~K}$ before decreasing in magnitude at higher temperatures. The characteristic field of the maximum is also weakly temperature-dependent, decreasing by approximately 0.05 tesla as the temperature is raised from 100 $\mathrm{mK}$ to $1.4 \mathrm{~K}$.

The behaviour of the real part of $\chi$ shown in Fig. 1 is suggestive of a line of first order transitions ending in a critical point at a temperature $T^{*}$ at which the susceptibility is maximised. The argument is as follows. At high temperatures, above that of the critical point, the susceptibility behaves like that of a crossover. As the temperature is decreased towards $T^{*}$, the peak susceptibility increases before being cut off by finite frequency or finite size effects. Below $T^{*}$, the dynamical response becomes sensitive to the physics of a first-order magnetic transition such as domain wall movement, causing the susceptibility to decrease. Previous work on systems such as $\left.\mathrm{Co}\left[\left(\mathrm{CH}_{3}\right)_{3} \mathrm{NH}\right] \mathrm{Cl}_{3} \cdot 2 \mathrm{H}_{2} \mathrm{O}\right]$ gives a precedent for this kind of behaviour, and also indicates that non-zero frequency

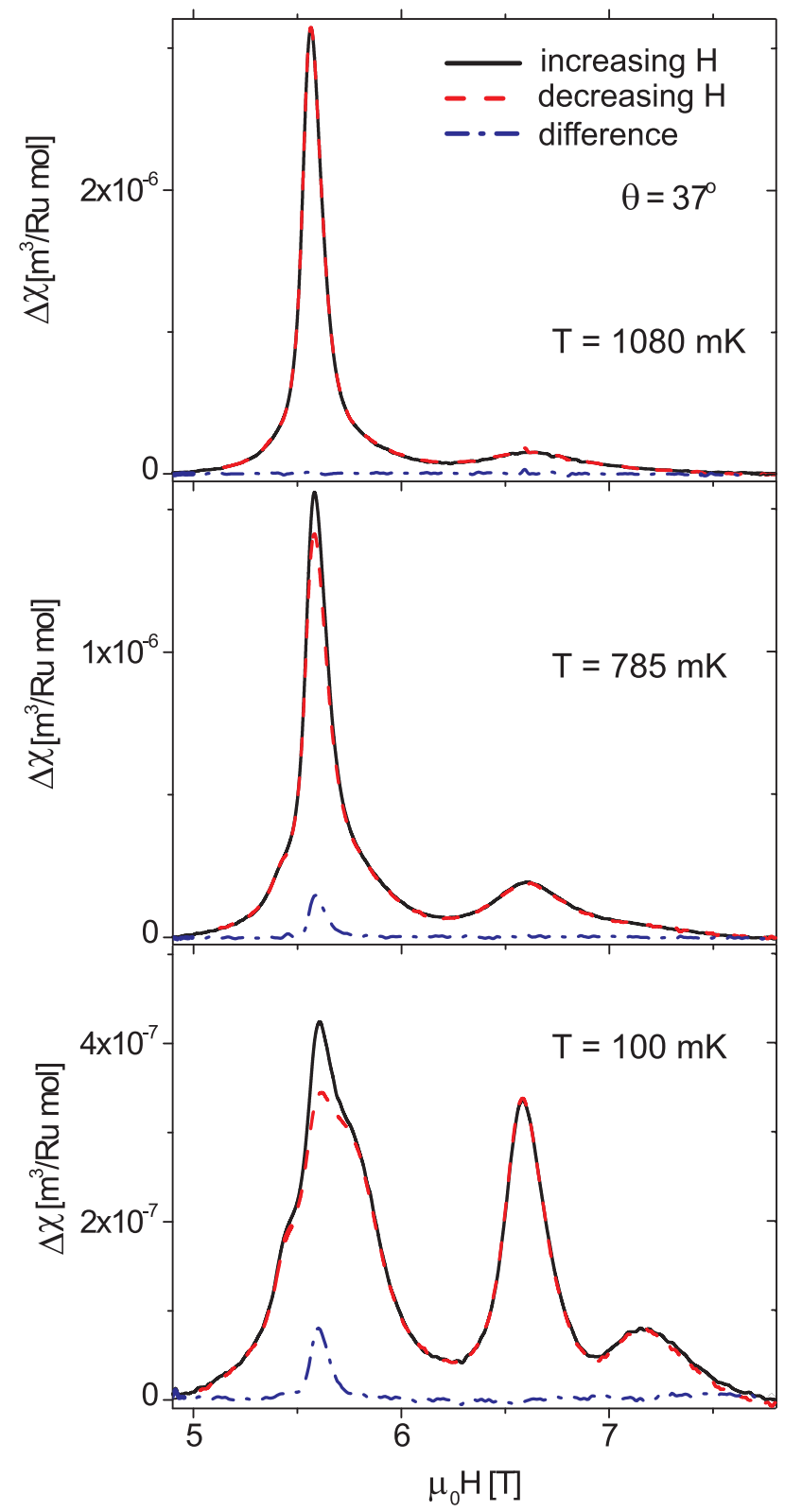

FIG. 2: (colour online) The real part of the differential magnetic susceptibility through the metamagnetic transition in $\mathrm{Sr}_{3} \mathrm{Ru}_{2} \mathrm{O}_{7}$. At $785 \mathrm{mK}$ and $100 \mathrm{mK}$, hysteresis is seen in the most prominent peak as the d.c. field, applied at approximately $40^{\circ}$ to the $a b$ plane, is increased (black solid line) and decreased (red dashed line). At $1080 \mathrm{mK}$ the hysteresis has completely disappeared. In each panel, the blue dot-dashed line gives the difference between the results for the increasing and decreasing field. In this case the frequency of the oscillating field was $63.54 \mathrm{~Hz}$. The data are quoted as $\Delta \chi$ because of the background subtraction procedure described in the text.

effects can play an important role even for low excitation frequencies of the kind $(\approx 80 \mathrm{~Hz})$ employed here 18 .

The above argument suggests that the data in Fig. 1 are consistent with the existence of a line of first-order transitions terminating in a critical point (for this field 


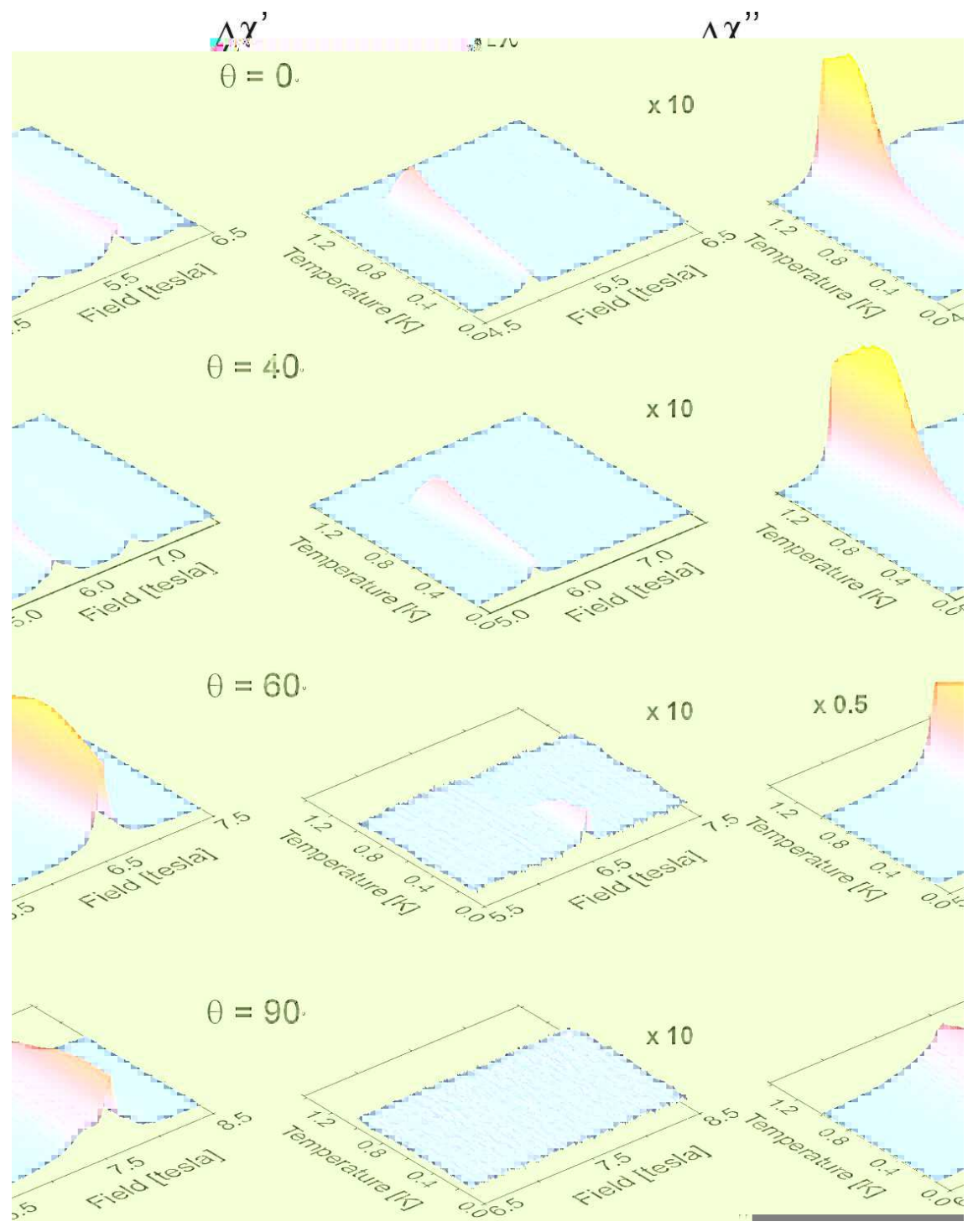

FIG. 3: (colour online) The real and imaginary parts $\left(\Delta \chi^{\prime}\right.$ and $\Delta \chi^{\prime \prime}$ respectively) of the differential susceptibility of $\mathrm{Sr}_{3} \mathrm{Ru}_{2} \mathrm{O}_{7}$ shown in a three-dimensional format with amplitude plotted against temperature and magnetic field, at a series of field angles. In all cases the frequency of measurement was $79.96 \mathrm{~Hz}$, and the data were taken in an increasing d.c. field. An excellent correlation is seen between the overall maximum in the real part of the susceptibility and the appearance at low temperatures of a peak in the imaginary part, indicative of the onset of dissipation. For the field applied parallel to the $c$ axis, the maximum is at the lowest temperature reached, and no peak at all is seen in the imaginary component. The imaginary parts of the susceptibility are multiplied by a factor of 10 , and at $\theta=60^{\circ}$ the real part by a factor 0.5 . 
angle) at approximately $1 \mathrm{~K}$, but they certainly cannot be regarded as proof of that hypothesis. Firmer evidence comes from a study of hysteresis, shown in Fig. 2. If the a.c. response of the system is studied while the main d.c. field is swept in opposite directions, a clear hysteretic response in the magnitude of $\Delta \chi$, but not in the value of $H$ at the peak, is seen for temperatures less than that at which the susceptibility is maximum ${ }^{19}$. The existence of hysteresis for temperatures below $T^{*}$ is the first strong support provided by our data to the idea of a first-order metamagnetic transition in this range of temperatures.

A second and related piece of evidence comes from consideration of a dissipative component in the a.c. signal itself. A wider sample of our total data set is shown in Fig. 3. In order to present the results of many field sweeps at each angle in a single plot, we adopt a colour-coded three dimensional format in which the magnitude of the dynamical susceptibility is plotted for an area of the $(H$, $T$ ) plane. Data are shown for field angles $\theta=0^{\circ}, 40^{\circ}, 60^{\circ}$ and $90^{\circ}$ from the $a b$-plane. In each case, the plots were constructed using field sweeps at discrete temperatures at approximately $100 \mathrm{mK}$ intervals from $100 \mathrm{mK}$ to the highest temperature shown. The second graph on the left of Fig. 3 therefore includes the data shown in Fig. 1 combined with the results of eight other field sweeps. The data for the real part of $\chi$ show a maximum susceptibility $\left(T^{*}, H^{*}\right)$ for which $T^{*}$ drops and $H^{*}$ rises as $\theta$ increases. For $\theta \gtrsim 80^{\circ}$, the highest observed value of $T^{*}$ is seen at the lowest temperature studied. The most significant aspect of this form of data presentation is that it gives a good visualisation of the correlation between the behaviour of the real part of the dynamical susceptibility and that of the imaginary part. Hysteresis of the kind shown in Fig. 2 would be expected to be accompanied by dissipation, and dissipation leads to the appearance of a feature in the imaginary part of an a.c. response. As can be seen from Fig. 3, we observe a direct correlation between the characteristic temperature of the maximum in the real part of the dynamical susceptibility and that below which a dissipation peak appears in the imaginary part.

It is hard to explain data of the kind shown in Fig. 3 without postulating the existence of a first-order metamagnetic transition below the empirically-defined characteristic temperature $T^{*}$. Before discussing the meaning of the characteristic temperature and field $\left(T^{*}, H^{*}\right)$ further, we present some more empirical findings relating to their angle dependence. The data shown in Fig. 3 are again only a sample from the complete data set acquired during our experiment, in which data were taken at a series of angles between $0^{\circ}$ and $90^{\circ}$ and at temperatures from $1400 \mathrm{mK}$ to $50 \mathrm{mK}$. A three-dimensional representation of the experimental phase diagram is given in Fig. 4. In this figure, the shaded surface is defined as the locus of points at which a peak is observed in the imaginary part of the dynamic susceptibility. Since this peak is linked to the dissipative response of the system, our interpretation of the shaded area is a surface of first order metamag-

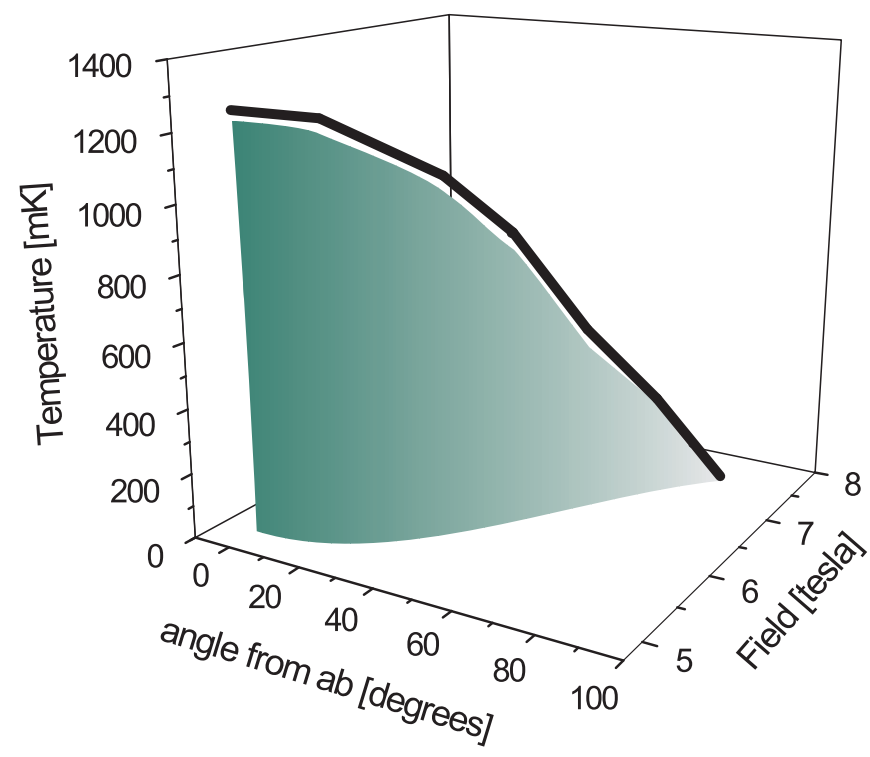

FIG. 4: (colour online) The phase diagram inferred from the whole body of susceptibility measurements reported in this paper. The shaded region represents the locus of points at which the imaginary part of the susceptibility has a peak, while the solid line is the locus of the overall maxima seen in the real part. Increasing magnetic field sweeps through the metamagnetic transition were taken at least every $100 \mathrm{mK}$, at a series of field angles as defined by the discrete points in Fig. 5.

netic phase transitions in the three-dimensional $(H, T$, $\theta$ ) space. The solid line was obtained from the series of points at which the real part of the susceptibility has its overall maximum (clearly visible in each of the panels on the left-hand side of Fig. 3), which we will argue below to be a line of metamagnetic critical points.

Fig. 4 is useful as an overall summary of our main experimental findings, but it can be difficult to read off some of the detailed information that has been obtained. For this reason, we present two projections from the figure in the main parts of Figs. 5 and 6 . In Fig. 5 we show the projection of the 'line of critical points' onto the $(T$, $\theta$ ) plane, showing how the characteristic temperature $T^{*}$ is depressed as the field angle is changed. In the main part of Fig. 6 the line is projected onto the $(H, \theta)$ plane, giving a representation of the angle dependence of the critical metamagnetic field. The inset to Fig. 5 shows that although the shaded surface in Fig. 4 is almost vertical, the slight curvature that exists is 'convex'. Here the points show the $\left(T^{*}, H^{*}\right)$ values of the overall maximum of the real part of $\chi$ at each of the two angles. The lines are two cuts from the plane at constant $\theta$ values of $50^{\circ}$ and $60^{\circ}$, so the inset confirms that, like the peak in the real part of $\chi$ (e.g. Fig. 1), the peak in the imaginary part falls to slightly lower field as temperature increases. The inset to Fig. 6 will be described in the next section. 


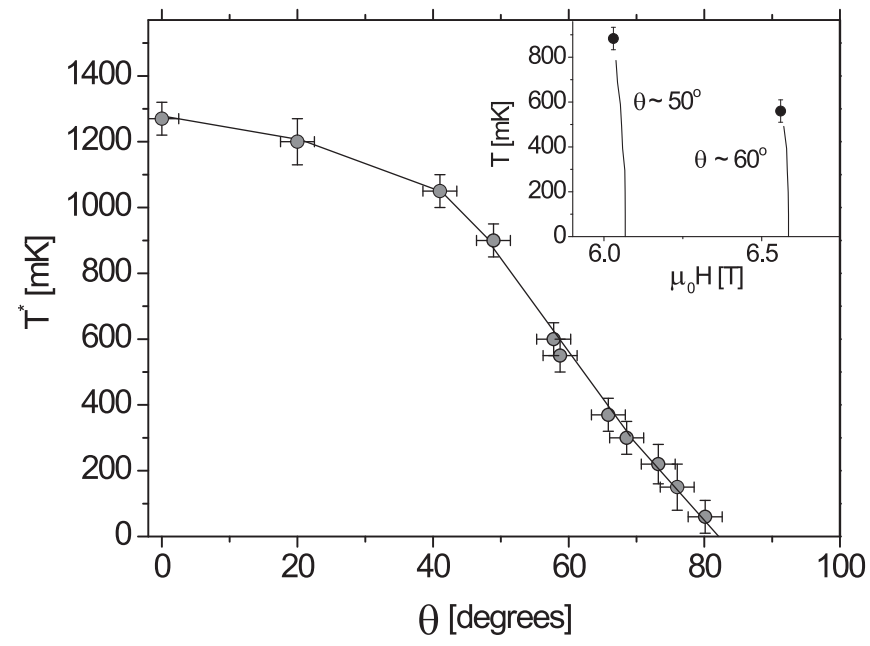

FIG. 5: The critical temperature $T^{*}$ (defined in the text) as a function of angle. The data are a discrete projection of the solid line from Fig. 4 onto the temperature - angle plane. For angles above $\approx 80^{\circ}$ the susceptibility is a monotonically increasing function to the lowest temperatures measured $(50 \mathrm{mK})$. The inset shows the field and temperature dependence at angles of $50^{\circ}$ and $60^{\circ}$ of the peak in the imaginary part of $\chi$, which we interpret as defining lines of first order transitions (solid lines). The solid points are the position in temperature and field of the overall maximum of the real part of $\chi$ at these angles.

\section{DISCUSSION}

The principal findings of our work, summarised in Figs. $1-6$, are two-fold. First, we believe that our results give very good evidence for the existence of a surface of first-order metamagnetic phase transitions in $\mathrm{Sr}_{3} \mathrm{Ru}_{2} \mathrm{O}_{7}$. Varying the combination of field and field angle terminates this surface at different temperatures $T^{*}$, with $T^{*}$ falling from approximately $1.25 \mathrm{~K}$ for $H \| a b$ to less than our base temperature of $50 \mathrm{mK}$ for $H \| c$. Second, the fact that field angle plays the role of a continuous tuning parameter for this system is a significant finding, which should open the way to future experiments involving fine adjustment of the balance between thermal and quantum fluctuations. One example of the effects of such a balance seems already to be observable in the data. The fall in the first-order metamagnetic transition field with increasing temperature (inset to Fig. 5) is in contrast with observations on other ferromagnetic itinerant metamagnets with higher critical temperatures 13 and with the predictions of a classical model20; the disordering effect of thermal fluctuations would imply that higher fields are needed to reach the state of enhanced magnetisation. This apparent paradox is no longer present when both thermal and quantum fluctuations are taken into account $\stackrel{\underline{9}}{ }$. At very low temperatures the latter dominate and the tendency is reversed.

The basic conclusions reached here about the behaviour of $T^{*}$ are also consistent with previous work on

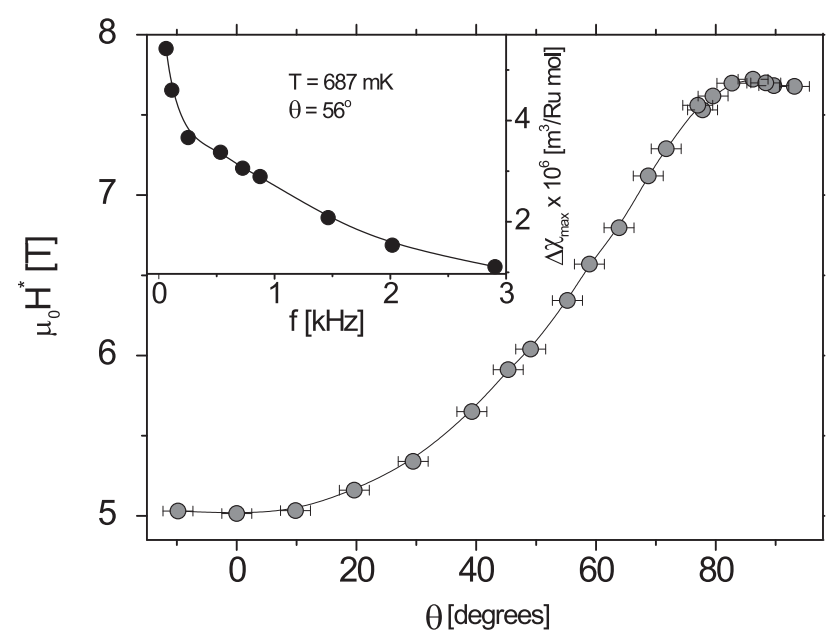

FIG. 6: The critical field $H^{*}$ (defined in the text) as a function of angle. The data are a discrete projection of the solid line from Fig. 4 onto the field - angle plane. The inset shows the frequency dependence of the peak in the real part of $\chi$ for $T^{*}$ at a field angle of $56^{\circ}$.

this material. For $H \| c$, transport measurements show a steep increase of the $A$ coefficient which measures the strength of the quadratic term in the resistivity. This suggests a diverging quasiparticle mass as the metamagnetic field is approached at low temperatures, as would be the case if there were a very low or zero temperature (quantum) metamagnetic critical point ${ }^{7}$.

A puzzle from the transport study was the appearance of anomalous powers higher than two in the $T$-dependent resistivity in a very narrow range of fields near the metamagnetic field ${ }^{7}$. In the absence of other data, one possibility for this anomalous behaviour was that the system was entering a region of first-order physics in this field range ${ }^{7.21}$. The data presented in Fig. 4 give no support to this idea. On the contrary, the unusual transport properties are seen in precisely a region of the phase diagram where we see no susceptibility evidence for firstorder transitions.

The data discussed so far give convincing evidence that a temperature, field and field- angle-dependent critical point must exist in $\mathrm{Sr}_{3} \mathrm{Ru}_{2} \mathrm{O}_{7}$, because the surface of first-order transitions that has been identified must end in a line of critical points. Open questions remain, however, about the precise nature of these critical points. A central issue concerning metamagnetic criticality is whether a simple picture based around longwavelength 'ferromagnetic' fluctuations ${ }^{9.20}$ gives an adequate description of the physics. For instance, it has been argued that even though a surface of first-order transitions can be observed by a long-wavelength probe like the magnetic susceptibility employed here, it is possible that its end-point might involve criticality of fluctuations at a much shorter wavelength ${ }^{16}$. The metamagnetic crossover in $\mathrm{CeRu}_{2} \mathrm{Si}_{2}$ has been argued to originate from such 'antiferromagnetic-like' fluctuations ${ }^{15}$, and nesting- 
related short-wavelength fluctuations have been observed by inelastic neutron scattering in $\mathrm{Sr}_{3} \mathrm{Ru}_{2} \mathrm{O}_{7}{ }_{7}^{22}$.

At first sight, our susceptibility data do not appear to favour the simple long-wavelength picture $\mathrm{e}^{\underline{\underline{9}}}$, because although an overall maximum is observed in the real part of $\chi$ at $T^{*}$, the divergence that such a model would predict is not seen. In real systems, divergences near phase transitions are cut off by finite size effects, disorder and (near magnetic transitions) demagnetisation ${ }^{18}$. We have made estimates of the magnitude of each of these effects, and have concluded that none of them would change a putative divergence to the much weaker feature shown in Fig. 3. However, we have discovered that although low, the finite frequency of these differential susceptibility measurements has a much stronger effect than we had anticipated. In the inset to Fig. 6, we show the frequency dependence of the peak in the real part of $\chi$ at $T^{*}$ (approximately $700 \mathrm{mK}$ ) for a field angle of approximately $56^{\circ}$. The peak height is seen to be rising steeply through the frequency range including that $(\approx 80 \mathrm{~Hz})$ of most of our measurements. Strong frequency dependences are also seen below $T^{*}$, although with a different functional form (data not shown). Although the origin is magnetic in both cases, there are differences. Below $T^{*}$ domain wall motion plays a dominant role, whereas at and above $T^{*}$, critical fluctuations are the main source. Although the sample studied here is very pure in comparison to most metallic oxides, the disorder that does exist may be playing a role in this unexpectedly strong frequency dependence ${ }^{23}$. The critical slowing down expected in the vicinity of a critical point can be greatly enhanced by the presence of disorder, with even very small quantities of it acting as 'pinning centres' for fluctuations. This pinning is in turn enhanced by the (low) scale of temperature at which the transitions take place.

The strength of the frequency dependence shown in the inset to Fig. 6 means that detailed analyses based on the field or temperature dependence of the amplitude of the data shown in Figs. $1-3$ must be done with great care. The data that we have presented are a fixed frequency snap-shot of a more complicated overall situation, and that snap-shot is not a good approximation to the zero frequency limit. We therefore believe that no firm conclusions can be drawn based on the weakness of the overall maximum seen in the real part of $\chi$. In particular, one certainly cannot rule out a genuine divergence in the long wavelength limit on the basis of a study such as this.

To our knowledge, previous studies of metamagnetism using finite frequency a.c. methods have been relatively rare. Most reports of susceptibility have been obtained from the results of measurements of quasi-d.c. magnetisation (in these studies the sweep rate of the d.c. field determines the characteristic time constant $)^{10,13}$. The work reported here emphasises that a.c. methods are a very sensitive means of detecting first-order behaviour and its temperature dependence $\underline{\underline{18}}$. They provide, in addition to the simplicity of their implementation, a way to study dynamical properties of the system. It is clear that a more thorough study of frequency dependence would be desirable. Preliminary measurements have shown that the determination of $T^{*}$ itself has some frequency dependence, which must be taken into account if elevated frequencies in the $\mathrm{kHz}$ range are employed. Below 100 $\mathrm{Hz}$, however, the effect is weak, so that we believe that the phase diagram presented in Fig. 4 is a good approximation to the results that would be obtained in the zero frequency limit.

Inspection of Fig. 4 shows that the inferred line of critical points passes below $50 \mathrm{mK}$ at an angle of approximately $80^{\circ}$, with a gradient that suggests that it would reach $T=0$ at approximately $85^{\circ}$ rather than $90^{\circ}$. There is no a priori reason to favour one angle over the other, or to assume that the critical point sits at $T=0$ only at one angle and not at a range of angles. On the basis of the data presented here, we cannot draw a firm conclusion either way. We also note that it may never be possible to do so. If the anomalous transport behaviour reported very near $H^{*}$ for $H \| c$ is due to the system entering some new state in the vicinity of the quantum critical point, the zero temperature critical point itself may not prove to be experimentally accessible. We plan more work in this area.

All of the discussion so far has concentrated on the most prominent peak seen in Figs. 1-3. No first-order behaviour is associated with either of the smaller peaks seen at higher fields for low $\theta$. On the basis of the data presented here, there is no reason to state that either of this peaks correspond to magnetic quantum critical points. Rather, the rapid disappearance with $\theta$ makes interpretation in terms of crossovers more likely. However, a detailed study of the frequency dependence will be necessary to settle this issue one way or the other.

Finally, we briefly raise the issue of the origin of the angular dependence. So far, we have concentrated on establishing the empirical fact that field angle acts as a continuous tuning parameter for the metamagnetism of $\mathrm{Sr}_{3} \mathrm{Ru}_{2} \mathrm{O}_{7}$ rather than discussing why that should be the case. Our results show that the metamagnetic transition presents both quantitative and qualitative changes as a function of the field angle that are not compatible with a simple projection of the magnetic field into the principal axes. It is known that lattice distortions have a pronounced effect on the magnetic properties of ruthenates ${ }^{26.27}$, and that in particular the metamagnetic transition in $\mathrm{Sr}_{3} \mathrm{Ru}_{2} \mathrm{O}_{7}$ is strongly affected by the application of hydrostatic and uniaxial pressure ${ }^{24,25}$. All this points towards magnetostriction effects being an important factor in the mechanisms of the metamagnetic transition in $\mathrm{Sr}_{3} \mathrm{Ru}_{2} \mathrm{O}_{7}$. Such effects are known to play an important role in the metamagnetism of other materials ${ }^{28}$. If there is a non-trivial angle-dependent magnetostriction associated with the metamagnetic transition in $\mathrm{Sr}_{3} \mathrm{Ru}_{2} \mathrm{O}_{7}$, there would be no reason for the effects of a changing field angle to be felt only as a projection. A puzzling feature of the magnetism of 
this material has been the fact that although there is some anisotropy of the metamagnetism, the low- temperature weak-field magnetic susceptibility is remarkably isotropio 17 . A scenario in which the anisotropy of the metamagnetism is related to magnetostriction promoted by higher applied fields might give a natural explanation for these apparently contradictory observations. A further appealing aspect of this picture is a natural analogy between changing field angle and changing pressure. In this sense the empirically determined phase diagram shown in Fig. 4 would have a close relation to the surface of pressure-induced metamagnetic phase transitions that results from Ginzburg-Landau treatments of varying sophistication 8.13 .29 .

\section{CONCLUSIONS}

In summary, we have used measurements of the differential dynamic susceptibility to demonstrate that changing magnetic field angle represents a continuous tuning parameter for the metamagnetism of $\mathrm{Sr}_{3} \mathrm{Ru}_{2} \mathrm{O}_{7}$. The empirical phase diagram contains a surface of first-order metamagnetic transitions whose end-point temperature $T^{*}$ can be tuned from $1.25 \mathrm{~K}$ for fields parallel to the $a b$ plane to below $50 \mathrm{mK}$ for fields applied parallel to the $c$ axis. These and previous transport studies are consistent with the existence of a quantum critical end-point for the latter field orientation. The work shows that a.c. susceptibility can be a sensitive and powerful probe of the classical- quantum crossover in metamagnetism, but that a fuller study of the frequency dependence is desirable.

\section{Acknowledgments}

We are grateful to L.M. Galvin for contributions to the early stages of this project, and to P. Coleman, E. M. Forgan, G.G. Lonzarich, A.J. Millis, A. Rosch, P. Riedi, S. Sachdev and A.J. Schofield for useful discussions. We thank the Leverhulme Trust, the UK EPSRC and the Royal Society for their support of the work.
1 J. A. Hertz, Phys. Rev. B 14, 1165 (1996).

2 A. J. Millis, Phys. Rev. B 487183 (1993).

3 N.D. Mathur, F. M. Grosche, S. R. Julian, I. R.Walker, D. M. Freye, R. K. W. Haselwimmer and G. G. Lonzarich, Nature 394, 39 (1998).

4 S. Sachdev, Quantum Phase Transitions, (Cambridge University Press, 1999).

${ }^{5}$ G. R. Stewart, Rev. Mod. Phys. 73, 797-855 (2001).

${ }^{6}$ R. S. Perry, L. M. Galvin, S. A. Grigera, L. Capogna, A. J. Schofield, A. P. Mackenzie, M. Chiao, S. R. Julian, S. I. Ikeda, S. Nakatsuji, Y. Maeno and C. Pfleiderer, Phys. Rev. Lett. 86, 2661 (2001).

7 S. A. Grigera, R. S. Perry, A. J. Schofield, M. Chiao, S. R. Julian, G. G. Lonzarich, S. I. Ikeda, Y. Maeno, A. J. Millis and A. P. Mackenzie, Science 294, 329 (2001).

8 S. A. Grigera, A. P. Mackenzie, A. J. Schofield, S. R. Julian and G. G. Lonzarich, Int. J. Mod Phys B 16, 3258 (2002).

9 A. J. Millis, A. J. Schofield, G. G. Lonzarich and S. A. Grigera, Phys. Rev. Lett. 88217204 (2002).

10 E. Stryjewski and N. Giordano, Adv. Phys. 26, 487 (1977).

11 E. P. Wohlfarth and P. Rhodes, Philos. Mag. 7, 1817 (1962).

12 Second order transitions with 'metamagnetic' signatures are possible in metals with local moments and additional bands of conduction electrons, see T. Senthil, Subir Sachdev and Matthias Vojta, cond-mat/0209144

13 T. Goto, K. Fukamichi and H. Yamada, Physica B 300, 167 (2001).

14 N.V. Mushnikov, T. Goto, K. Kamishima, H. Yamada, A.V. Andreev, Y. Shiokawa, A. Iwao and V. Sechovsky, Phys. Rev. B 596677 (1999).

15 see for example J. Flouquet, P. Haen, S. Raymond, D. Aoki and G. Knebel, Phys. B 319 251-261 (2002) and references therein.

16 M. Chiao, C. Pfleiderer, R. Daou, A. McCollam, S. R. Julian, G. G. Lonzarich, R. S. Perry, A. P. Mackenzie and
Y. Maeno, preprint, cond-mat/0207697 (2002).

17 S. Ikeda, Y. Maeno, S. Nakatsuji, M. Kosaka and Y. Uwatoko, Phys. Rev. B 62, R6089 (2000).

18 A. J. Duyneveldt, J. Appl. Phys. 53, 8006 (1982); A. J. van Duyneveldt, in Proceedings of the NATO advanced research workshop on magnetic molecular materials, Vol. 198 of NATO Advanced Study Institute, Series E: Applied Sciences (Kluwer Academic, Dordrecht 1991) and references therein.

19 The hysteretic behaviour that we see with these a.c. techniques differs from that usually observed in metamagnetic transitions studied with d.c. techniques (See, for example ref 13). The a.c. measurements include effects due to the dynamics of the first order transition, which is markedly dependent on whether the change is between a state of low and high magnetisation or vice versa.

20 see for example H. Yamada, Phys. Rev. B 47, 11211 (1993).

21 G. Aeppli and F. Soh, Science 294, 329 (2001).

22 L. Capogna, E. M. Forgan, S. M. Hayden, A. Wildes, J. A. Duffy, A. P. Mackenzie, R. S. Perry, S. Ikeda, Y. Maeno, and S. P. Brown, preprint, cond-mat/0201556 (2002).

23 Very small amounts of disorder are known to play important roles in determining the critical behaviour close to a QCP, see for example A. Rosch Phys. Rev. Lett. 82, 4280 (1999).

24 M. Chiao, C. Pfleiderer, S. R. Julian, G. G. Lonzarich, R. S. Perry, A. P. Mackenzie and Y. Maeno, Physica B 312-313, 698 (2002).

25 Uniaxial stress applied along the $c$-axis can even drive $\mathrm{Sr}_{3} \mathrm{Ru}_{2} \mathrm{O}_{7}$ ferromagnetic below $100 \mathrm{~K}$. That is now thought to be the origin of the behaviour as a function of pressure first reported in Ref. 17; S. Ikeda, private communication.

${ }^{26}$ H. Shaked, J. D. Jorgensen, S. Short, O. Chmaissem, S.-I. Ikeda and Y. Maeno Phys. Rev. B 62, 8725 (2000).

27 O. Friedt, M. Braden, G. Andr, P. Adelmann, S. Nakatsuji and Y. Maeno, Phys. Rev. B 63, 174432 (2001). 
${ }^{28}$ P. Haen, J. Flouquet, F. Lapierre, P. Lejay and G. Re414, 427 (2001). menyi, J. Low Temp. Phys. 67, 391 (1987).

${ }^{29}$ C. Pfleiderer, S. R. Julian and G. G. Lonzarich, Nature 